Kragujevac Journal of Mathematics

Volume 45(1) (2021), Pages 103-114.

\title{
ON $\lambda$-PSEUDO BI-STARLIKE FUNCTIONS WITH RESPECT TO SYMMETRIC POINTS ASSOCIATED TO SHELL-LIKE CURVES
}

\author{
G. MURUGUSUNDARAMOORTHY ${ }^{1}$, K. VIJAYA ${ }^{1}$, AND H. ÖZLEM GÜNEY $^{2 *}$ \\ ABSTRACT. In this paper we define a new subclass $\lambda$-pseudo bi-starlike functions \\ with respect to symmetric points of $\Sigma$ related to shell-like curves connected with \\ Fibonacci numbers and determine the initial Taylor-Maclaurin coefficients $\left|a_{2}\right|$ and \\ $\left|a_{3}\right|$ for $f \in \mathcal{P S}_{\mathcal{L}}^{\lambda} \mathcal{S}_{s, \Sigma}^{\lambda}(\alpha, \tilde{p}(z))$. Further we determine the Fekete-Szegö result for the \\ function class $\mathcal{P S S}_{\mathcal{S}, \Sigma}^{\lambda}(\alpha, \tilde{p}(z))$ and for special cases, corollaries are stated which \\ some of them are new and have not been studied so far.
}

\section{INTRODUCTION}

Let $\mathcal{A}$ denote the class of functions $f$ which are analytic in the open unit disk $\mathbb{U}=\{z: z \in \mathbb{C}$ and $|z|<1\}$. Also, let $\mathcal{S}$ denote the class of functions in $\mathcal{A}$ which are univalent in $\mathbb{U}$ and normalized by the conditions $f(0)=f^{\prime}(0)-1=0$ and are of the form

$$
f(z)=z+\sum_{n=2}^{\infty} a_{n} z^{n} .
$$

The Koebe one quarter theorem [4] ensures that the image of $\mathbb{U}$ under every univalent function $f \in \mathcal{A}$ contains a disk of radius $\frac{1}{4}$. Thus every univalent function $f$ has an inverse $f^{-1}$ satisfying

$$
f^{-1}(f(z))=z,(z \in \mathbb{U}) \text { and } f\left(f^{-1}(w)\right)=w\left(|w|<r_{0}(f), r_{0}(f) \geq \frac{1}{4}\right) .
$$

A function $f \in \mathcal{A}$ is said to be bi-univalent in $\mathbb{U}$ if both $f$ and $f^{-1}$ are univalent in $\mathbb{U}$. Let $\Sigma$ denote the class of bi-univalent functions defined in the unit disk $\mathbb{U}$. Since

Key words and phrases. Analytic functions, bi-univalent, shell-like curve, Fibonacci numbers, starlike functions.

2010 Mathematics Subject Classification. Primary: 30C45. Secondary: 30C50.

DOI 10.46793/Kg.JMat2101.103M

Received: June 14, 2018.

Accepted: October 08, 2018. 
$f \in \Sigma$ has the Maclaurian series given by (1.1), a computation shows that its inverse $g=f^{-1}$ has the expansion

$$
g(w)=f^{-1}(w)=w-a_{2} w^{2}+\left(2 a_{2}^{2}-a_{3}\right) w^{3}+\cdots .
$$

One can see a short history and examples of functions in the class $\Sigma$ in [17]. Several authors have introduced and investigated subclasses of bi-univalent functions and obtained bounds for the initial coefficients (see [2,3,11,17-19]).

An analytic function $f$ is subordinate to an analytic function $F$ in $\mathbb{U}$, written as $f \prec F(z \in \mathbb{U})$, provided there is an analytic function $\omega$ defined on $\mathbb{U}$ with $\omega(0)=0$ and $|\omega(z)|<1$ satisfying $f(z)=F(\omega(z))$. It follows from Schwarz Lemma that

$$
f(z) \prec F(z) \Rightarrow f(0)=F(0) \text { and } f(\mathbb{U}) \subset F(\mathbb{U}), \quad z \in \mathbb{U}
$$

(for details see [4], [10]). We recall important subclasses of $\mathcal{S}$ in geometric function theory such that if $f \in \mathcal{A}$ and $\frac{z f^{\prime}(z)}{f(z)} \prec p(z)$ and $1+\frac{z f^{\prime \prime}(z)}{f^{\prime}(z)} \prec p(z)$, where $p(z)=\frac{1+z}{1-z}$, then we say that $f$ is starlike and convex, respectively. These functions form known classes denoted by $\mathcal{S}^{*}$ and $\mathcal{C}$, respectively. An interesting case when the function $p$ is convex but is not univalent was considered in [6]. Ma and Minda [9] unified various subclasses of starlike and convex functions for which either of the quantity $\frac{z f^{\prime}(z)}{f(z)}$ or $1+\frac{z f^{\prime \prime}(z)}{f^{\prime}(z)}$ is subordinate to a more general superordinate function. Here superordinate functions is an analytic function $\varphi$ with positive real part in the unit disc $\mathbb{U}$ with $\varphi(0)=1, \varphi^{\prime}(0)>0$ and it maps $\mathbb{U}$ onto a region starlike with respect to 1 and is symmetric with respect to the real axis. The class $S^{*}(\varphi)$ and $K(\varphi)$ denote Ma-Minda starlike and Ma-Minda convex functions, respectively. If we restrict considerations to the absorbing geometric shape of $p(\mathbb{U})$, is parabolic domain or an elliptic domain or in an interior of hyperbola, further the cases, when $p(\mathbb{U})$ is an interior of the right loop of the Lemniscate of Bernoulli or in leaf-like domain in recent past (see $[6,15,16]$ and also references cited therein) for the case of functions in $\mathcal{A}$. The behavior of the coefficients are unpredictable when the bi-univalency condition is imposed on the function $f \in \mathcal{A}$ in our present work we attempted to find initial coefficients for $f \in \Sigma$ by considering the geometric shape of $p(\mathbb{U})$ related to a shell-like curve connected with Fibonacci numbers.

Recently, in [14], Sokół introduced the class $\mathcal{S} \mathcal{L}$ of shell-like functions as the set of functions $f \in \mathcal{A}$ which is described in the following definition.

Definition 1.1. The function $f \in \mathcal{A}$ belongs to the class $\mathcal{S} \mathcal{L}$ if it satisfies the condition that

$$
\frac{z f^{\prime}(z)}{f(z)} \prec \tilde{p}(z)
$$

with $\tilde{p}(z)=\frac{1+\tau^{2} z^{2}}{1-\tau z-\tau^{2} z^{2}}$, where $\tau=(1-\sqrt{5}) / 2 \approx-0.618$.

It should be observed $\mathcal{S} \mathcal{L}$ is a subclass of the starlike functions $\mathcal{S}^{*}$.

The function $\tilde{p}$ is not univalent in $\mathbb{U}$, but it is univalent in the disc $|z|<(3-\sqrt{5}) / 2 \approx$ 0.38. For example, $\tilde{p}(0)=\tilde{p}(-1 / 2 \tau)=1$ and $\tilde{p}\left(e^{\mp i \arccos (1 / 4)}\right)=\sqrt{5} / 5$, and it may also 
be noticed that

$$
\frac{1}{|\tau|}=\frac{|\tau|}{1-|\tau|}
$$

which shows that the number $|\tau|$ divides $[0,1]$ such that it fulfils the golden section. The image of the unit circle $|z|=1$ under $\tilde{p}$ is a curve described by the equation given by

$$
(10 x-\sqrt{5}) y^{2}=(\sqrt{5}-2 x)(\sqrt{5} x-1)^{2},
$$

which is translated and revolved trisectrix of Maclaurin. The curve $\tilde{p}\left(r e^{i t}\right)$ is a closed curve without any loops for $0<r \leq r_{0}=(3-\sqrt{5}) / 2 \approx 0.38$. For $r_{0}<r<1$, it has a loop, and for $r=1$, it has a vertical asymptote. Since $\tau$ satisfies the equation $\tau^{2}=1+\tau$, this expression can be used to obtain higher powers $\tau^{n}$ as a linear function of lower powers, which in turn can be decomposed all the way down to a linear combination of $\tau$ and 1 . The resulting recurrence relationships yield Fibonacci numbers $u_{n}$ :

$$
\tau^{n}=u_{n} \tau+u_{n-1}
$$

In [13] Raina and Sokół showed that

$$
\begin{aligned}
\tilde{p}(z) & =\frac{1+\tau^{2} z^{2}}{1-\tau z-\tau^{2} z^{2}}=\left(t+\frac{1}{t}\right) \frac{t}{1-t-t^{2}} \\
& =\frac{1}{\sqrt{5}}\left(t+\frac{1}{t}\right)\left(\frac{1}{1-(1-\tau) t}-\frac{1}{1-\tau t}\right) \\
& =\left(t+\frac{1}{t}\right) \sum_{n=1}^{\infty} u_{n} t^{n}=1+\sum_{n=1}^{\infty}\left(u_{n-1}+u_{n+1}\right) \tau^{n} z^{n}
\end{aligned}
$$

where

$$
u_{n}=\frac{(1-\tau)^{n}-\tau^{n}}{\sqrt{5}}, \quad \tau=\frac{1-\sqrt{5}}{2}, \quad n=1,2, \ldots
$$

This shows that the relevant connection of $\tilde{p}$ with the sequence of Fibonacci numbers $u_{n}$, such that $u_{0}=0, u_{1}=1, u_{n+2}=u_{n}+u_{n+1}$ for $n=0,1,2, \ldots$ And they got

$$
\begin{aligned}
\tilde{p}(z) & =1+\sum_{n=1}^{\infty} \tilde{p}_{n} z^{n} \\
& =1+\left(u_{0}+u_{2}\right) \tau z+\left(u_{1}+u_{3}\right) \tau^{2} z^{2}+\sum_{n=3}^{\infty}\left(u_{n-3}+u_{n-2}+u_{n-1}+u_{n}\right) \tau^{n} z^{n} \\
& =1+\tau z+3 \tau^{2} z^{2}+4 \tau^{3} z^{3}+7 \tau^{4} z^{4}+11 \tau^{5} z^{5}+\cdots .
\end{aligned}
$$

Let $\mathcal{P}(\beta), 0 \leq \beta<1$, denote the class of analytic functions $p$ in $\mathbb{U}$ with $p(0)=1$ and $\operatorname{Re}\{p(z)\}>\beta$. Especially, we will use $\mathcal{P}$ instead of $\mathcal{P}(0)$.

Theorem $1.1([7])$. The function $\tilde{p}(z)=\frac{1+\tau^{2} z^{2}}{1-\tau z-\tau^{2} z^{2}}$ belongs to the class $\mathcal{P}(\beta)$ with $\beta=\sqrt{5} / 10 \approx 0.2236$.

Now we give the following lemma which will use in proving. 
Lemma $1.1([12])$. Let $p \in \mathcal{P}$ with $p(z)=1+c_{1} z+c_{2} z^{2}+\cdots$, then $\left|c_{n}\right| \leq 2$, for $n \geq 1$.

\section{Bi-Univalent Function Class $\mathcal{P S}_{\mathcal{S}}^{\lambda} \mathcal{L}_{s, \Sigma}^{\lambda}(\alpha, \tilde{p}(z))$}

In this section, we introduce a new subclass of $\Sigma$ associated with $\lambda$-pseudo bistarlike functions with respect to symmetric points related to shell-like curves connected with Fibonacci numbers and obtain the initial Taylor coefficients $\left|a_{2}\right|$ and $\left|a_{3}\right|$ for the function class by subordination.

The class $\mathcal{L}_{\lambda}(\gamma)$ of $\lambda$-pseudo-starlike functions of order $\gamma(0 \leq \gamma<1)$ were introduced and investigated by Babalola [1] whose geometric conditions satisfy

$$
\operatorname{Re}\left(\frac{z\left(f^{\prime}(z)\right)^{\lambda}}{f(z)}\right)>\gamma .
$$

He showed that all pseudo-starlike functions are Bazilevič of type $\left(1-\frac{1}{\lambda}\right)$ order $\gamma^{\frac{1}{\lambda}}$ and univalent in open unit disk $\mathbb{U}$. If $\lambda=1$, we have the class of starlike functions of order $\gamma$, which in this context, are 1 -pseudo-starlike functions of order $\gamma$. Babalola [1] remarked that though for $\lambda>1$, these classes of $\lambda$-pseudo starlike functions clone the analytic representation of starlike functions, it is not yet known the possibility of any inclusion relations between them.

Motivated by the works of Dziok et al. in [7] on the class of convex and $\alpha$-convex functions related to a shell-like curve connected with Fibonacci numbers, Eker et al. and [5] on bi-pseudo-starlike functions class and obtained the initial coefficients $\left|a_{2}\right|$ and $\left|a_{3}\right|$ with respect to other points in this paper we define the new class named as $\lambda$-pseudo bi-starlike functions with respect to symmetric points related to shell-like curves connected with Fibonacci numbers as follows.

Definition 2.1. Let $0 \leq \alpha \leq 1$ and $\lambda \geq 1$ is real. A function $f \in \Sigma$ of the form (1.1) is said to be in the class $\mathcal{P S} \mathcal{L}_{s, \Sigma}^{\lambda}(\alpha, \tilde{p}(z))$ if the following subordination hold:

$$
(1-\alpha) \frac{2 z\left(f^{\prime}(z)\right)^{\lambda}}{f(z)-f(-z)}+\alpha \frac{2\left[\left(z\left(f^{\prime}(z)\right)\right)^{\prime}\right]^{\lambda}}{[f(z)-f(-z)]^{\prime}} \prec \tilde{p}(z)
$$

and

$$
(1-\alpha) \frac{2 w\left(g^{\prime}(w)\right)^{\lambda}}{g(w)-g(-w)}+\alpha \frac{2\left[\left(w\left(g^{\prime}(w)\right)\right)^{\prime}\right]^{\lambda}}{[g(w)-g(-w)]^{\prime}} \prec \tilde{p}(w)
$$

where $\tau=(1-\sqrt{5}) / 2 \approx-0.618$ where $z, w \in \mathbb{U}$ and $g$ is given by (1.2).

Specializing the parameter $\lambda=1$ we have the following definitions, respectively.

Definition 2.2. A function $f \in \Sigma$ of the form (1.1) is said to be in the class $\mathcal{P S} \mathcal{L}_{s, \Sigma}^{1}(\alpha, \tilde{p}(z)) \equiv \mathcal{M} \mathcal{S} \mathcal{L}_{s, \Sigma}(\alpha, \tilde{p}(z))$ if the following subordination hold:

$$
(1-\alpha) \frac{2 z f^{\prime}(z)}{f(z)-f(-z)}+\alpha \frac{2\left(z\left(f^{\prime}(z)\right)\right)^{\prime}}{[f(z)-f(-z)]^{\prime}} \prec \tilde{p}(z)
$$


and

$$
(1-\alpha) \frac{2 w g^{\prime}(w)}{g(w)-g(-w)}+\alpha \frac{2\left(w\left(g^{\prime}(w)\right)\right)^{\prime}}{[g(w)-g(-w)]^{\prime}} \prec \tilde{p}(w)
$$

where $\tau=(1-\sqrt{5}) / 2 \approx-0.618$ where $z, w \in \mathbb{U}$ and $g$ is given by (1.2).

Definition 2.3. A function $f \in \Sigma$ of the form (1.1) is said to be in the class $\mathcal{P S} \mathcal{L}_{s, \Sigma}^{1}(0, \tilde{p}(z)) \equiv \mathcal{S} \mathcal{L}_{s, \Sigma}^{*}(\tilde{p}(z))$ if the following subordination hold:

$$
\frac{2 z f^{\prime}(z)}{f(z)-f(-z)} \prec \tilde{p}(z)
$$

and

$$
\frac{2 w g^{\prime}(w)}{g(w)-g(-w)} \prec \tilde{p}(w)
$$

where $\tau=(1-\sqrt{5}) / 2 \approx-0.618$ where $z, w \in \mathbb{U}$ and $g$ is given by (1.2).

Definition 2.4. A function $f \in \Sigma$ of the form (1.1) is said to be in the class $\mathcal{P S} \mathcal{L}_{s, \Sigma}^{1}(1, \tilde{p}(z)) \equiv \mathcal{K} \mathcal{L}_{s, \Sigma}(\tilde{p}(z))$ if the following subordination hold:

$$
\frac{2\left(z\left(f^{\prime}(z)\right)\right)^{\prime}}{[f(z)-f(-z)]^{\prime}} \prec \tilde{p}(z)
$$

and

$$
\frac{2\left(w\left(g^{\prime}(w)\right)\right)^{\prime}}{[g(w)-g(-w)]^{\prime}} \prec \tilde{p}(w),
$$

where $\tau=(1-\sqrt{5}) / 2 \approx-0.618$, where $z, w \in \mathbb{U}$ and $g$ is given by (1.2).

In the following theorem we determine the initial Taylor coefficients $\left|a_{2}\right|$ and $\left|a_{3}\right|$ for the function class $\mathcal{P S} \mathcal{L}_{s, \Sigma}^{\lambda}(\alpha, \tilde{p}(z))$. Later we will reduce these bounds to other classes for special cases as corollaries which are new and have not been studied sofar.

Theorem 2.1. Let $f$ given by (1.1) be in the class $\mathcal{P S}_{\mathcal{L}}^{\lambda}{ }_{s, \Sigma}^{\lambda}(\alpha, \tilde{p}(z))$. Then

$$
\left|a_{2}\right| \leq \frac{|\tau|}{\sqrt{4 \lambda^{2}(1+\alpha)^{2}-\left\{2 \lambda^{2}\left(6 \alpha^{2}+9 \alpha+5\right)-\lambda+2 \alpha+1\right\} \tau}}
$$

and

$$
\left|a_{3}\right| \leq \frac{2 \lambda|\tau|\left[2 \lambda(1+\alpha)^{2}-\left\{(\lambda-1)(1+3 \alpha)-6 \lambda(1+\alpha)^{2}\right\} \tau\right]}{(3 \lambda-1)(1+2 \alpha)\left[4 \lambda^{2}(1+\alpha)^{2}-\left\{2 \lambda^{2}\left(6 \alpha^{2}+9 \alpha+5\right)-\lambda+2 \alpha+1\right\} \tau\right]}
$$

Proof. Firstly, let $p(z)=1+p_{1} z+p_{2} z^{2}+\cdots$, and $p \prec \tilde{p}$. Then there exists an analytic function $u$ such that $u(0)=0 ;|u(z)|<1$ in $\mathbb{U}$ and $p(z)=\tilde{p}(u(z))$. Therefore, the function

$$
h(z)=\frac{1+u(z)}{1-u(z)}=1+c_{1} z+c_{2} z^{2}+\cdots
$$

is in the class $\mathcal{P}$. It follows that

$$
u(z)=\frac{c_{1} z}{2}+\left(c_{2}-\frac{c_{1}^{2}}{2}\right) \frac{z^{2}}{2}+\left(c_{3}-c_{1} c_{2}+\frac{c_{1}^{3}}{4}\right) \frac{z^{3}}{2}+\cdots
$$


and

$$
\begin{aligned}
\tilde{p}(u(z))= & +\frac{\tilde{p}_{1} c_{1} z}{2}+\left\{\frac{1}{2}\left(c_{2}-\frac{c_{1}^{2}}{2}\right) \tilde{p}_{1}+\frac{c_{1}^{2}}{4} \tilde{p}_{2}\right\} z^{2} \\
& +\left\{\frac{1}{2}\left(c_{3}-c_{1} c_{2}+\frac{c_{1}^{3}}{4}\right) \tilde{p}_{1}+\frac{1}{2} c_{1}\left(c_{2}-\frac{c_{1}^{2}}{2}\right) \tilde{p}_{2}+\frac{c_{1}^{3}}{8} \tilde{p}_{3}\right\} z^{3}+\cdots
\end{aligned}
$$

And similarly, there exists an analytic function $v$ such that $v(0)=0 ;|v(w)|<1$ in $\mathbb{U}$ and $p(w)=\tilde{p}(v(w))$. Therefore, the function

$$
k(w)=\frac{1+v(w)}{1-v(w)}=1+d_{1} w+d_{2} w^{2}+\cdots
$$

is in the class $\mathcal{P}$. It follows that

$$
v(w)=\frac{d_{1} w}{2}+\left(d_{2}-\frac{d_{1}^{2}}{2}\right) \frac{w^{2}}{2}+\left(d_{3}-d_{1} d_{2}+\frac{d_{1}^{3}}{4}\right) \frac{w^{3}}{2}+\cdots
$$

and

$$
\begin{aligned}
\tilde{p}(v(w))= & +\frac{\tilde{p}_{1} d_{1} w}{2}+\left\{\frac{1}{2}\left(d_{2}-\frac{d_{1}^{2}}{2}\right) \tilde{p}_{1}+\frac{d_{1}^{2}}{4} \tilde{p}_{2}\right\} w^{2} \\
& +\left\{\frac{1}{2}\left(d_{3}-d_{1} d_{2}+\frac{d_{1}^{3}}{4}\right) \tilde{p}_{1}+\frac{1}{2} d_{1}\left(d_{2}-\frac{d_{1}^{2}}{2}\right) \tilde{p}_{2}+\frac{d_{1}^{3}}{8} \tilde{p}_{3}\right\} w^{3}+\cdots
\end{aligned}
$$

Let $f \in \mathcal{P S} \mathcal{L}_{s, \Sigma}^{\lambda}(\alpha, \tilde{p}(z))$ and $g=f^{-1}$. Considering (2.1) and (2.2), we have

$$
(1-\alpha) \frac{2 z\left(f^{\prime}(z)\right)^{\lambda}}{f(z)-f(-z)}+\alpha \frac{2\left[\left(z\left(f^{\prime}(z)\right)\right)^{\prime}\right]^{\lambda}}{[f(z)-f(-z)]^{\prime}}=\tilde{p}(u(z))
$$

and

$$
(1-\alpha) \frac{2 w\left(g^{\prime}(w)\right)^{\lambda}}{g(w)-g(-w)}+\alpha \frac{2\left[\left(w\left(g^{\prime}(w)\right)\right)^{\prime}\right]^{\lambda}}{[g(w)-g(-w)]^{\prime}}=\tilde{p}(v(w)),
$$

where $\tau=(1-\sqrt{5}) / 2 \approx-0.618, z, w \in \mathbb{U}$ and $g$ is given by (1.2). Since

$$
\begin{aligned}
& (1-\alpha) \frac{2 z\left(f^{\prime}(z)\right)^{\lambda}}{f(z)-f(-z)}+\alpha \frac{2\left[\left(z\left(f^{\prime}(z)\right)\right)^{\prime}\right]^{\lambda}}{[f(z)-f(-z)]^{\prime}} \\
= & 1+2 \lambda(1+\alpha) a_{2} z+\left[2 \lambda(\lambda-1)(1+3 \alpha) a_{2}^{2}+(3 \lambda-1)(1+2 \alpha) a_{3}\right] z^{2}+\cdots
\end{aligned}
$$

and

$$
\begin{aligned}
& (1-\alpha) \frac{2 w\left(g^{\prime}(w)\right)^{\lambda}}{g(w)-g(-w)}+\alpha \frac{2\left[\left(w\left(g^{\prime}(w)\right)\right)^{\prime}\right]^{\lambda}}{[g(w)-g(-w)]^{\prime}} \\
= & 1-2 \lambda(1+\alpha) a_{2} w+\left\{\left[2\left(\lambda^{2}+2 \lambda-1\right)+2 \alpha\left(3 \lambda^{2}+3 \lambda-2\right)\right] a_{2}^{2}\right. \\
& \left.\left.-(3 \lambda-1)(1+2 \alpha) a_{3}\right]\right\} w^{2}+\cdots .
\end{aligned}
$$


Thus, we have

$$
\begin{aligned}
& 1+2 \lambda(1+\alpha) a_{2} z+\left[2 \lambda(\lambda-1)(1+3 \alpha) a_{2}^{2}+(3 \lambda-1)(1+2 \alpha) a_{3}\right] z^{2}+\cdots \\
= & 1+\frac{\tilde{p}_{1} c_{1} z}{2}+\left[\frac{1}{2}\left(c_{2}-\frac{c_{1}^{2}}{2}\right) \tilde{p}_{1}+\frac{c_{1}^{2}}{4} \tilde{p}_{2}\right] z^{2} \\
& +\left[\frac{1}{2}\left(c_{3}-c_{1} c_{2}+\frac{c_{1}^{3}}{4}\right) \tilde{p}_{1}+\frac{1}{2} c_{1}\left(c_{2}-\frac{c_{1}^{2}}{2}\right) \tilde{p}_{2}+\frac{c_{1}^{3}}{8} \tilde{p}_{3}\right] z^{3}+\cdots
\end{aligned}
$$

and

$$
\begin{aligned}
& 1-2 \lambda(1+\alpha) a_{2} w \\
& \left.+\left\{\left[2\left(\lambda^{2}+2 \lambda-1\right)+2 \alpha\left(3 \lambda^{2}+3 \lambda-2\right)\right] a_{2}^{2}-(3 \lambda-1)(1+2 \alpha) a_{3}\right]\right\} w^{2}+\ldots \\
= & 1+\frac{\tilde{p}_{1} d_{1} w}{2}+\left[\frac{1}{2}\left(d_{2}-\frac{d_{1}^{2}}{2}\right) \tilde{p}_{1}+\frac{d_{1}^{2}}{4} \tilde{p}_{2}\right] w^{2} \\
& +\left[\frac{1}{2}\left(d_{3}-d_{1} d_{2}+\frac{d_{1}^{3}}{4}\right) \tilde{p}_{1}+\frac{1}{2} d_{1}\left(d_{2}-\frac{d_{1}^{2}}{2}\right) \tilde{p}_{2}+\frac{d_{1}^{3}}{8} \tilde{p}_{3}\right] w^{3}+\cdots .
\end{aligned}
$$

It follows from (2.4) and (2.5) that

$$
2 \lambda(1+\alpha) a_{2}=\frac{c_{1} \tau}{2}
$$

$$
2 \lambda(\lambda-1)(1+3 \alpha) a_{2}^{2}+(3 \lambda-1)(1+2 \alpha) a_{3}=\frac{1}{2}\left(c_{2}-\frac{c_{1}^{2}}{2}\right) \tau+\frac{c_{1}^{2}}{4} 3 \tau^{2}
$$

and

$$
\begin{aligned}
& -2 \lambda(1+\alpha) a_{2}=\frac{d_{1} \tau}{2}, \\
& {\left[2\left(\lambda^{2}+2 \lambda-1\right)+2 \alpha\left(3 \lambda^{2}+3 \lambda-2\right)\right] a_{2}^{2}-(3 \lambda-1)(1+2 \alpha) a_{3} } \\
= & \frac{1}{2}\left(d_{2}-\frac{d_{1}^{2}}{2}\right) \tau+\frac{d_{1}^{2}}{4} 3 \tau^{2} .
\end{aligned}
$$

From (2.6) and (2.8), we have

$$
c_{1}=-d_{1}
$$

and

$$
a_{2}^{2}=\frac{\left(c_{1}^{2}+d_{1}^{2}\right)}{32 \lambda^{2}(1+\alpha)^{2}} \tau^{2}
$$

Hence,

$$
\left|a_{2}\right| \leq \frac{|\tau|}{2 \lambda(1+\alpha)}
$$

Now, by summing (2.7) and (2.9), we obtain

$$
\left[2\left(2 \lambda^{2}+\lambda-1\right)+4 \alpha\left(3 \lambda^{2}-1\right)\right] a_{2}^{2}=\frac{1}{2}\left(c_{2}+d_{2}\right) \tau-\frac{1}{4}\left(c_{1}^{2}+d_{1}^{2}\right) \tau+\frac{3}{4}\left(c_{1}^{2}+d_{1}^{2}\right) \tau^{2} .
$$


Substituting (2.10) in (2.11), we have

$$
4\left[4 \lambda^{2}(1+\alpha)^{2}-\left\{2 \lambda^{2}\left(6 \alpha^{2}+9 \alpha+5\right)-\lambda+2 \alpha+1\right\} \tau\right] a_{2}^{2}=\left(c_{2}+d_{2}\right) \tau^{2} .
$$

Therefore, by Lemma (1.1) we obtain

$$
\left|a_{2}\right| \leq \frac{|\tau|}{\sqrt{4 \lambda^{2}(1+\alpha)^{2}-\left\{2 \lambda^{2}\left(6 \alpha^{2}+9 \alpha+5\right)-\lambda+2 \alpha+1\right\} \tau}} .
$$

It is clear that

$$
\begin{aligned}
& \min \left\{\frac{|\tau|}{2 \lambda(1+\alpha)}, \frac{|\tau|}{\sqrt{4 \lambda^{2}(1+\alpha)^{2}-\left\{2 \lambda^{2}\left(6 \alpha^{2}+9 \alpha+5\right)-\lambda+2 \alpha+1\right\} \tau}}\right\} \\
= & \frac{|\tau|}{\sqrt{4 \lambda^{2}(1+\alpha)^{2}-\left\{2 \lambda^{2}\left(6 \alpha^{2}+9 \alpha+5\right)-\lambda+2 \alpha+1\right\} \tau}} .
\end{aligned}
$$

So, we obtain the inequality (2.3).

Now, so as to find the bound on $\left|a_{3}\right|$, let's subtract from (2.7) and (2.9). So, we find

$$
2(3 \lambda-1)(1+2 \alpha) a_{3}-2(3 \lambda-1)(1+2 \alpha) a_{2}^{2}=\frac{1}{2}\left(c_{2}-d_{2}\right) \tau .
$$

Hence, we get

$$
2(3 \lambda-1)(1+2 \alpha)\left|a_{3}\right| \leq 2|\tau|+2(3 \lambda-1)(1+2 \alpha)\left|a_{2}\right|^{2} .
$$

Then, in view of (2.13), we obtain

$$
\left|a_{3}\right| \leq \frac{2 \lambda|\tau|\left[2 \lambda(1+\alpha)^{2}-\left\{(\lambda-1)(1+3 \alpha)-6 \lambda(1+\alpha)^{2}\right\} \tau\right]}{(3 \lambda-1)(1+2 \alpha)\left[4 \lambda^{2}(1+\alpha)^{2}-\left\{2 \lambda^{2}\left(6 \alpha^{2}+9 \alpha+5\right)-\lambda+2 \alpha+1\right\} \tau\right]}
$$

Taking $\lambda=1$, in the above theorem, we have the following the initial Taylor coefficients $\left|a_{2}\right|$ and $\left|a_{3}\right|$ for the function classes $\mathcal{M} \mathcal{S} \mathcal{L}_{s, \Sigma}(\alpha, \tilde{p}(z))$.

Corollary 2.1. Let $f$ given by (1.1) be in the class $\mathcal{M} S \mathcal{L}_{s, \Sigma}(\alpha, \tilde{p}(z))$. Then

$$
\left|a_{2}\right| \leq \frac{|\tau|}{\sqrt{4(1+\alpha)^{2}-2\left(6 \alpha^{2}+10 \alpha+5\right) \tau}}
$$

and

$$
\left|a_{3}\right| \leq \frac{2(1+\alpha)^{2}|\tau|(1-3 \tau)}{(1+2 \alpha)\left[4(1+\alpha)^{2}-2\left(6 \alpha^{2}+10 \alpha+5\right) \tau\right]} .
$$

Further by taking $\alpha=0$ and $\alpha=1$ in Corollary 2.1, we have the following the initial Taylor coefficients $\left|a_{2}\right|$ and $\left|a_{3}\right|$ for the function classes $\mathcal{S L}_{s, \Sigma}^{*}(\alpha, \tilde{p}(z))$ and $\mathcal{K} \mathcal{L}_{s, \Sigma}(\alpha, \tilde{p}(z))$, respectively.

Corollary 2.2. Let $f$ given by $(1.1)$ be in the class $\mathcal{S}_{s, \Sigma}^{*}(\alpha, \tilde{p}(z))$. Then

$$
\left|a_{2}\right| \leq \frac{|\tau|}{\sqrt{4-10 \tau}}
$$


and

$$
\left|a_{3}\right| \leq \frac{|\tau|(1-3 \tau)}{2-5 \tau}
$$

Corollary 2.3. Let $f$ given by $(1.1)$ be in the class $\mathcal{K} \mathcal{L}_{s, \Sigma}(\alpha, \tilde{p}(z))$. Then

$$
\left|a_{2}\right| \leq \frac{|\tau|}{\sqrt{16-42 \tau}}
$$

and

$$
\left|a_{3}\right| \leq \frac{4|\tau|(1-3 \tau)}{3(8-21 \tau)}
$$

3. Fekete-Szegö inequality for the Function Class $\mathcal{P S}_{\mathcal{S}}^{\lambda} \mathcal{L}_{s, \Sigma}^{\lambda}(\alpha, \tilde{p}(z))$

Fekete and Szegö [8] introduced the generalized functional $\left|a_{3}-\mu a_{2}^{2}\right|$, where $\mu$ is some real number. Due to Zaprawa [20], in the following theorem we determine the Fekete-Szegö functional for $f \in \mathcal{P S} \mathcal{L}_{s, \Sigma}^{\lambda}(\alpha, \tilde{p}(z))$.

Theorem 3.1. Let $f$ given by (1.1) be in the class $\mathcal{P S}_{s, \Sigma}^{\lambda}(\alpha, \tilde{p}(z))$ and $\mu \in \mathbb{R}$. Then we have

$$
\left|a_{3}-\mu a_{2}^{2}\right| \leq \begin{cases}\frac{|\tau|}{(3 \lambda-1)(1+2 \alpha)}, & 0 \leq|h(\mu)| \leq \frac{|\tau|}{4(3 \lambda-1)(1+2 \alpha)} \\ 4|h(\mu)|, & |h(\mu)| \geq \frac{|\tau|}{4(3 \lambda-1)(1+2 \alpha)}\end{cases}
$$

where

$$
h(\mu)=\frac{(1-\mu) \tau^{2}}{4\left[4 \lambda^{2}(1+\alpha)^{2}-\left\{2 \lambda^{2}\left(6 \alpha^{2}+9 \alpha+5\right)-\lambda+2 \alpha+1\right\} \tau\right]} .
$$

Proof. From (2.12) and (2.14) we obtain

$$
\begin{aligned}
& a_{3}-\mu a_{2}^{2} \\
& +\frac{(1-\mu)\left(c_{2}+d_{2}\right) \tau^{2}}{4\left[4 \lambda^{2}(1+\alpha)^{2}-\left\{2 \lambda^{2}\left(6 \alpha^{2}+9 \alpha+5\right)-\lambda+2 \alpha+1\right\} \tau\right]}+\frac{\tau\left(c_{2}-d_{2}\right)}{4(3 \lambda-1)(1+2 \alpha)} \\
= & \left(\frac{(1-\mu) \tau^{2}}{4\left[4 \lambda^{2}(1+\alpha)^{2}-\left\{2 \lambda^{2}\left(6 \alpha^{2}+9 \alpha+5\right)-\lambda+2 \alpha+1\right\} \tau\right]} \frac{\tau}{4(3 \lambda-1)(1+2 \alpha)}\right) c_{2} \\
& +\left(\frac{(1-\mu) \tau^{2}}{4\left[4 \lambda^{2}(1+\alpha)^{2}-\left\{2 \lambda^{2}\left(6 \alpha^{2}+9 \alpha+5\right)-\lambda+2 \alpha+1\right\} \tau\right]} \frac{\tau}{4(3 \lambda-1)(1+2 \alpha)}\right) d_{2} .
\end{aligned}
$$

So, we have

$$
a_{3}-\mu a_{2}^{2}=\left(h(\mu)+\frac{|\tau|}{4(3 \lambda-1)(1+2 \alpha)}\right) c_{2}+\left(h(\mu)-\frac{|\tau|}{4(3 \lambda-1)(1+2 \alpha)}\right) d_{2}
$$

where

$$
h(\mu)=\frac{(1-\mu) \tau^{2}}{4\left[4 \lambda^{2}(1+\alpha)^{2}-\left\{2 \lambda^{2}\left(6 \alpha^{2}+9 \alpha+5\right)-\lambda+2 \alpha+1\right\} \tau\right]} .
$$


Then, by taking modulus of (3.1), we conclude that

$$
\left|a_{3}-\mu a_{2}^{2}\right| \leq \begin{cases}\frac{|\tau|}{(3 \lambda-1)(1+2 \alpha)}, & 0 \leq|h(\mu)| \leq \frac{|\tau|}{4(3 \lambda-1)(1+2 \alpha)} \\ 4|h(\mu)|, & |h(\mu)| \geq \frac{|\tau|}{4(3 \lambda-1)(1+2 \alpha)}\end{cases}
$$

Taking $\mu=1$, we have the following corollary.

Corollary 3.1. If $f \in \mathcal{P S} \mathcal{L}_{s, \Sigma}^{\lambda}(\alpha, \tilde{p}(z))$, then

$$
\left|a_{3}-a_{2}^{2}\right| \leq \frac{|\tau|}{(3 \lambda-1)(1+2 \alpha)} .
$$

If we can take the parameter $\lambda=1$ in Theorem 3.1, we can state the following.

Corollary 3.2. Let $f$ given by (1.1) be in the class $\mathcal{M} \mathcal{S} \mathcal{L}_{s, \Sigma}(\alpha, \tilde{p}(z))$ and $\mu \in \mathbb{R}$. Then we have

where

$$
\left|a_{3}-\mu a_{2}^{2}\right| \leq \begin{cases}\frac{|\tau|}{2(1+2 \alpha)}, & 0 \leq|h(\mu)| \leq \frac{|\tau|}{8(1+2 \alpha)} \\ 4|h(\mu)|, & |h(\mu)| \geq \frac{|\tau|}{8(1+2 \alpha)}\end{cases}
$$

$$
h(\mu)=\frac{(1-\mu) \tau^{2}}{4\left[4(1+\alpha)^{2}-2\left\{6 \alpha^{2}+10 \alpha+5\right\} \tau\right]} .
$$

Further by taking $\alpha=0$ and $\alpha=1$ in the above corollary, we have the following the Fekete-Szegö inequalities for the function classes $\mathcal{S L}_{s, \Sigma}^{*}(\alpha, \tilde{p}(z))$ and $\mathcal{K} \mathcal{L}_{s, \Sigma}(\alpha, \tilde{p}(z))$, respectively.

Corollary 3.3. Let $f$ given by (1.1) be in the class $\mathcal{S} \mathcal{L}_{s, \Sigma}^{*}(\alpha, \tilde{p}(z))$ and $\mu \in \mathbb{R}$. Then we have

where

$$
\left|a_{3}-\mu a_{2}^{2}\right| \leq \begin{cases}\frac{|\tau|}{2}, & 0 \leq|h(\mu)| \leq \frac{|\tau|}{8} \\ 4|h(\mu)|, & |h(\mu)| \geq \frac{|\tau|}{8}\end{cases}
$$

$$
h(\mu)=\frac{(1-\mu) \tau^{2}}{8[2-5 \tau]} .
$$

Corollary 3.4. Let $f$ given by (1.1) be in the class $\mathcal{K} \mathcal{L}_{s, \Sigma}(\alpha, \tilde{p}(z))$ and $\mu \in \mathbb{R}$. Then we have

where

$$
\left|a_{3}-\mu a_{2}^{2}\right| \leq \begin{cases}\frac{|\tau|}{6}, & 0 \leq|h(\mu)| \leq \frac{|\tau|}{24} \\ 4|h(\mu)|, & |h(\mu)| \geq \frac{|\tau|}{24}\end{cases}
$$

$$
h(\mu)=\frac{(1-\mu) \tau^{2}}{8[8-21 \tau]} .
$$


Acknowledgements. We record our sincere thanks to the referees for their valuable comments to improve the paper in present form.

\section{REFERENCES}

[1] K. O. Babalola, On $\lambda$-pseudo-starlike functions, J. Class. Anal. 3(2) (2013), 137-147.

[2] D. A. Brannan, J. Clunie and W. E. Kirwan, Coefficient estimates for a class of star-like functions, Canad. J. Math. 22 (1970), 476-485.

[3] D. A. Brannan and T. S. Taha, On some classes of bi-univalent functions, Studia Univ. BabesBolyai Math. 31(2) (1986), 70-77.

[4] P. L. Duren, Univalent functions, in: Grundlehren der Mathematischen Wissenschaften, Band 259, Springer-Verlag, New York, Berlin, Heidelberg, Tokyo, 1983.

[5] S. S. Eker and B. Seker, On $\lambda$-pseudo bi-starlike and $\lambda$-pseudo bi-convex functions with respect to symmetrical points, Tbilisi Math. J. 11(1) (2018), 49-57.

[6] R. Jurasiska and J. Stankiewicz, Coefficients in some classes defined by subordination to multivalent majorants, in: Proceedings of Conference on Complex Analysis, Bielsko-Biala, 2001, Ann. Polon. Math. 80 (2003), 163-170.

[7] J. Dziok, R. K. Raina and J. Sokół, On $\alpha$-convex functions related to a shell-like curve connected with Fibonacci numbers, Appl. Math. Comp. 218 (2011), 996-1002.

[8] M. Fekete and G. Szegö, Eine Bemerkung über ungerade schlichte Functionen, J. Lond. Math. Soc. 8 (1933), 85-89.

[9] W. Ma and D. Minda, A Unified treatment of some special cases of univalent functions, in: Proceedings of the Conference on Complex Analysis, Tianjin, 1992, International Press, Cambridge, USA, 157-169.

[10] S. S. Miller and P. T. Mocanu, Differential Subordinations Theory and Applications, Series of Monographs and Text Books in Pure and Applied Mathematics 225, Marcel Dekker, New York, 2000.

[11] M. Lewin, On a coefficient problem for bi-univalent functions, Proc. Amer. Math. Soc. 18 (1967), 63-68.

[12] Ch. Pommerenke, Univalent Functions, Vandenhoeck and Ruprecht, Göttingen, 1975.

[13] R. K. Raina and J. Sokół, Fekete-Szegö problem for some starlike functions related to shell-like curves, Math. Slovaca 66 (2016), 135-140.

[14] J. Sokół, On starlike functions connected with Fibonacci numbers, Folia Scient. Univ. Tech. Resoviensis 175 (1999), 111-116.

[15] J. Sokół, On some subclass of strongly starlike functions, Demonstr. Math. 31(1) (1998), 81-86

[16] J. Sokół and J. Stankiewicz, Radius of convexity of some subclasses of strongly starlike functions, Folia Scient. Univ. Tech. Resoviensis 147 (1996), 101-105

[17] H. M. Srivastava, A. K. Mishra and P. Gochhayat, Certain subclasses of analytic and bi-univalent functions, Appl. Math. Lett. 23(10) (2010), 1188-1192.

[18] Q.-H. Xu, Y.-C. Gui and H. M. Srivastava, Coefficinet estimates for a certain subclass of analytic and bi-univalent functions, Appl. Math. Lett. 25 (2012), 990-994.

[19] X-F. Li and A-P Wang, Two new subclasses of bi-univalent functions, International Mathematical Forum 7(30) (2012), 1495-1504.

[20] P. Zaprawa, On the Fekete-Szegö problem for classes of bi-univalent functions, Bull. Belg. Math. Soc. Simon Stevin 21(10) (2014),169-178. 
${ }^{1}$ School of Advanced Sciences, Vellore Institute of TeChlogy, VELLORE -632014, INDIA

Email address: gmsmoorthy@yahoo.com

${ }^{1}$ School of Advanced Sciences, Vellore Institute of TeChlogy, VELLORE -632014, INDIA

Email address: kvijaya@vit.ac.in

${ }^{2}$ Dicle University, Faculty of Science, Department of MATHEMATics, DIYARBAKIR, TURKEY

Email address: ozlemg@dicle.edu.tr

*Corresponding Author 